\title{
Clarification to article Meier R., Panzica M. First Results with a Resorbable MgYREZr Compression Screw in Unstable Scaphoid Fractures Show Extensive Bone Cysts
}

Handchir Mikrochir Plast Chir 2017; 49: 37-41; DOI: 10.1055/s-0042-121416

The responsible ethics commission was not consulted. Accordingly, approval by an ethics commission is not available. Therefore, according to the current state of knowledge, this publication is based on a case series, rather than on a prospective study as initially stated.

Karl-Josef Prommersberger, Editor

Reinhard Meier, Author 